\title{
Backward Recursion in Layered Space-Time Non-linear Interference Cancellation Detectors
}

\author{
W. C. Freitas Jr., D. C. Moreira, C. C. Cavalcante and A. L. F. de Almeida,
}

\begin{abstract}
In this letter we present a new approach for non-linear interference cancellation detectors allowing all layers of a generic layered space-time multiplexing scheme to achieve an improved diversity order at the receiver which enhances its performance as a whole. This goal is achieved by adding a backward recursion in traditional non-linear interference cancellation detectors, e.g. successive interference cancellation (SIC) and ordered successive interference cancellation (OSIC). Our illustrative results confirm that by using this simple detection approach, all layers of a generic layered space-time multiplexing scheme achieve an improved diversity gain at the receiver.
\end{abstract}

Index Terms-Multiple antenna systems, successive interference cancellation (SIC), backward recursion

\section{INTRODUCTION}

$\mathbf{M}$ IMO ANTENNA SYSTEMS have been a topic of intense research during the last decade due to their capability to provide high data rates and robustness to wireless links. Multiple input multiple output (MIMO) is considered a mandatory technology to be employed in the upcoming wireless communication systems, such as $3 \mathrm{G}+$ and $4 \mathrm{G}$, for achieving the envisaged quality of service (QoS) levels [1].

Despite the well-known trade-off between diversity and multiplexing whenever a MIMO structure is considered [2], the goal of maximizing the data rate is achieved by means of spatial multiplexing schemes. Contrary to diversity schemes, where most of the signal processing is performed at the transmitter, multiplexing schemes use the receiver as the processing unit for interference cancellation purposes [3]. Typical solutions are zero forcing (ZF) and minimum mean square error (MMSE) receivers for the linear spatial filtering, and successive interference cancellation (SIC) as non-linear interference cancellation scheme [4]. Although very simple, the SIC receiver suffers from the problem of error propagation since the first detected layer must have a good temporal coding. Further, during detection, the initial layers have lower diversity orders, since the amount of interference is proportional to the the number of non-detected layers at this point [4].

In order to improve detection performance, some works have proposed iterative methods for processing the information and extracting more diversity during the receiver signal processing for interference cancellation. The method of [5] performs an iterative maximal ratio combining (MRC)-vector after the interference cancellation stage in order to improve

The authors are with the Wireless Telecommunications Research Group (GTEL), Department of Teleinformatics Engineering (DETI) Federal University of Ceará (UFC), Fortaleza-CE, Brazil, e-mails: (\{walter,darlan,charles, andre $\} @$ gtel.ufc.br), Phone/Fax: +55-85-33669470 performance over the traditional spatial multiplexing scheme, also known as vertical bell-labs layered space-time (VBLAST) structure.

In this letter, we propose the use of a backward recursion in layered space-time non-linear interference cancellation detectors in order to provide improved diversity order at the receiver to all layers. The idea is quite simple and shows potential due its performance gains over classical SIC and ordered successive interference cancellation (OSIC) detectors.

The rest of this paper is organized as follows. In Section II the system model is described. The rationale of the layered detection is discussed in Section III while Section IV is devoted to the explanation of the simple backward processing idea for improving the performance of layered transmission. Simulation results corroborating the merits of the proposed receiver processing are shown in Section V. The paper is concluded in Section VI.

\section{Channel And System Models}

We consider a transmitter equipped with an $M$-element antenna array and a receiver equipped with an $N$-element antenna array $(N \geq M)$. The wireless channel matrix is assumed to have rich scattering and undergo flat-fading. The fading between each transmit and receive antenna pair is assumed to be independent. A quasi-static block fading model is assumed. Furthermore, the total transmit power is fixed and equally divided across the transmit antennas. Ideal symbol timing and pulse shaping are assumed at the transmitter and receiver. Thus, we can relate the transmit and receive symbols in a given symbol period in complex baseband form as:

$$
\mathbf{x}=\sqrt{\frac{E_{s}}{M}} \mathbf{H} \mathbf{s}+\mathbf{v},
$$

where $\mathbf{H}$ denotes the $N \times M$ channel matrix, $\mathbf{x}$ denotes the complex received vector of dimension $N \times 1$, s denotes the transmitted symbol vector having dimension $M \times 1, \mathrm{v}$ is the additive white circularly symmetric complex gaussian noise samples with zero mean and variance $\sigma_{\mathrm{v}}^{2}=N_{0} / 2$ per dimension and $E_{s} / M$ is the energy of a symbol radiated from each transmit antenna.

\section{LAYERED SPACE-TIME INTERFERENCE CANCELlation DETECTORS}

In order to separate the signals received from the several transmit antennas at the receiver, interference cancellation detection algorithms should be considered to detect the co-channel signals. Initially, detection techniques were proposed in the literature based on the conventional linear 
nulling, which consider one layer as the desired, one per turn, and the other ones are treated as interferers. The nulling vectors in each turn are calculated following a given criterion such as ZF and MMSE [6].

In $\mathrm{ZF}$ criterion the decision for the $i$-th layer is made nulling the other $M-1$ layers, and the nulling spatial filter $\mathbf{W}$ is given by

$$
\mathbf{W}^{\mathrm{H}}=\sqrt{\frac{M}{E_{s}}} \mathbf{H}^{\dagger}=\sqrt{\frac{M}{E_{s}}}\left(\mathbf{H}^{\mathrm{H}} \mathbf{H}\right)^{-1} \mathbf{H}^{\mathrm{H}},
$$

where $\mathbf{H}^{\dagger}$ is the Moore-Penrose generalized inverse matrix [7] and $(\cdot)^{\mathrm{H}}$ is the Hermitian operator.

Considering the MMSE criterion the nulling spatial filter $\mathbf{W}$ is found minimizing the following cost function

$$
J_{\mathrm{MMSE}}=E\left\{\left\|\mathbf{W}^{\mathrm{H}} \mathbf{x}-\mathbf{s}\right\|^{2}\right\},
$$

such that

$$
\mathbf{W}^{\mathrm{H}}=\sqrt{\frac{M}{E_{s}}}\left[\mathbf{H}^{\mathrm{H}} \mathbf{H}+\sigma_{\mathbf{v}}^{2} \mathbf{I}_{M}\right]^{-1} \mathbf{H}^{\mathrm{H}},
$$

where $\mathbf{I}_{M}$ is an $M \times M$ identity matrix. As in the $\mathrm{ZF}$ criterion, the output vector for the $i$-th layer of the MMSE detector is $y_{i}$ given by

$$
y_{i}=\mathbf{w}_{i}^{\mathrm{H}} \mathbf{x}
$$

where $\mathbf{w}_{i}^{\mathrm{H}}$ is the vector associated with the $i$-th row of the matrix W. Both linear nulling detectors, ZF and MMSE, could improve their performance if an additional step is considered after the nulling of interference [6].

A superior detector performance for layered space-time MIMO schemes can be reached if the contribution of the detected layers to the the received signal is reconstructed and cancelled. Assuming correct decisions (ideal case without error propagation), the resulting signal is free from the interference of the layers already detected, yielding better estimates of the remaining symbols, which improves the whole performance of the detector. This detector is known in the literature as SIC or nulling and cancelling detector.

In SIC, the layers are detected sequentially. Initially, the received signal $\mathrm{x}$ goes through a nulling detector for the first layer (we can apply ZF or MMSE criteria), whose output is used to produce a hard estimate of the symbols at this layer, $\hat{s}_{1}$. Then, the contribution of the first layer to the received signal is estimated and cancelled, generating the signal $\mathbf{x}_{2}$. This process is recursive until the last spatial layer is reached. In general, at the $i$-th layer, the signal $\mathbf{x}_{i}$, hopefully free from the interference of layers $1, \cdots, i-1$, goes through a nulling detector that tries to mitigate the interference from layers $i+$ $1, \cdots, M$. A hard estimate of the symbol at this layer, $\hat{s}_{i}$, is then produced, based on the output of the nulling detector. Then, the contribution of this layer to the "received signal" $\mathbf{x}_{i}$ is estimated and cancelled. This procedure yields a modified received signal given by

$$
\mathbf{x}_{i+1}=\mathbf{x}_{i}-\hat{s}_{i} \mathbf{h}_{i},
$$

where $\mathbf{h}_{i}$ is the $i$-th column of the channel matrix $\mathbf{H}$ corresponding to the channel gains associated to layer $i$, and $\hat{s}_{i} \mathbf{h}_{i}$ represents the estimated interference from the $i$-th layer.
The result is that $\mathbf{x}_{i+1}$ is free from the interference coming from layers $1, \ldots, i$. This signal is then fed into the linear detector for the $(i+1)$-th layer and the process is repeated until layer $M$. The traditional SIC detector is represented in Figure 1 as the forward recursion stage.

Note that the performance of SIC can be improved if the layers are detected in an appropriate order, resulting in the OSIC algorithm [8]. Indeed, one of the disadvantages of SIC is that the signal associated with the first detected layer may exhibit a lower received signal-to-noise ratio (SNR) than some other layer. This may increase the probability of detection errors, which can propagate through the recursive detection process, degrading performance of the overall receiver. This problem can be mitigated if the layers are ordered by decreasing SNR, so that the first layer to be detected is that with the highest SNR.

\section{A. Performance of Layered Space-Time Interference Cancellation Detection}

In [9], the performance of MIMO linear MMSE nulling followed by SIC was evaluated. The authors of [9] derive simple expression to evaluate the performance of low-complexity SIC algorithm. Basically, the performance of each layer of a MIMO linear MMSE followed by SIC with uniform mean power over all the transmit antennas is equivalent to that of a MIMO linear MMSE nulling system with 1 transmit antenna and $N-M+i$ receive antennas, where the $(i+1)$-th layer is considered to be free from the interference coming from layers $1, \ldots, i$ [9].

Using this result, we can claim that the diversity order is increased for each layer in the MIMO linear MMSE nulling followed by SIC cancelling approach. Clearly, this is unfair to the first layers to be detected by the algorithm since these layers will not obtain the full diversity order at the receiver. In order to overcome this issue we propose the backward recursion technique in non-linear interference cancellation detectors. As an illustration we will consider the SIC detector using the MMSE nulling criterion and the VBLAST spatial multiplexing scheme.

\section{BACKWARD RECURSION IN SUCCESSIVE INTERFERENCE CANCELATION ALGORITHM}

In order to improve the performance of SIC-based detectors in layered space-time multiplexing schemes, we propose to extend the cancelling approach for the first layers in a backward recursive way. Using this simple idea the performance of the SIC-based detectors is improved since now all layers will experience an increased diversity order at the receiver due to the interference cancellation of the SIC approach. The proposed backward recursion algorithm is summarized in Algorithm 1 using a MMSE nulling spatial filter. However, this idea could be extended straightforwardly to the $\mathrm{ZF}$ receiver criterion.

In Algorithm 1, $Q(\cdot)$ denotes the quantization operation in accordance with the modulation in use, $\hat{s}_{i}$ is the estimation of the symbol of the $i$-th layer at the forward recursion stage and $\hat{s}_{i}^{\prime}$ is the estimation of the symbol of the $i$-th layer at the 


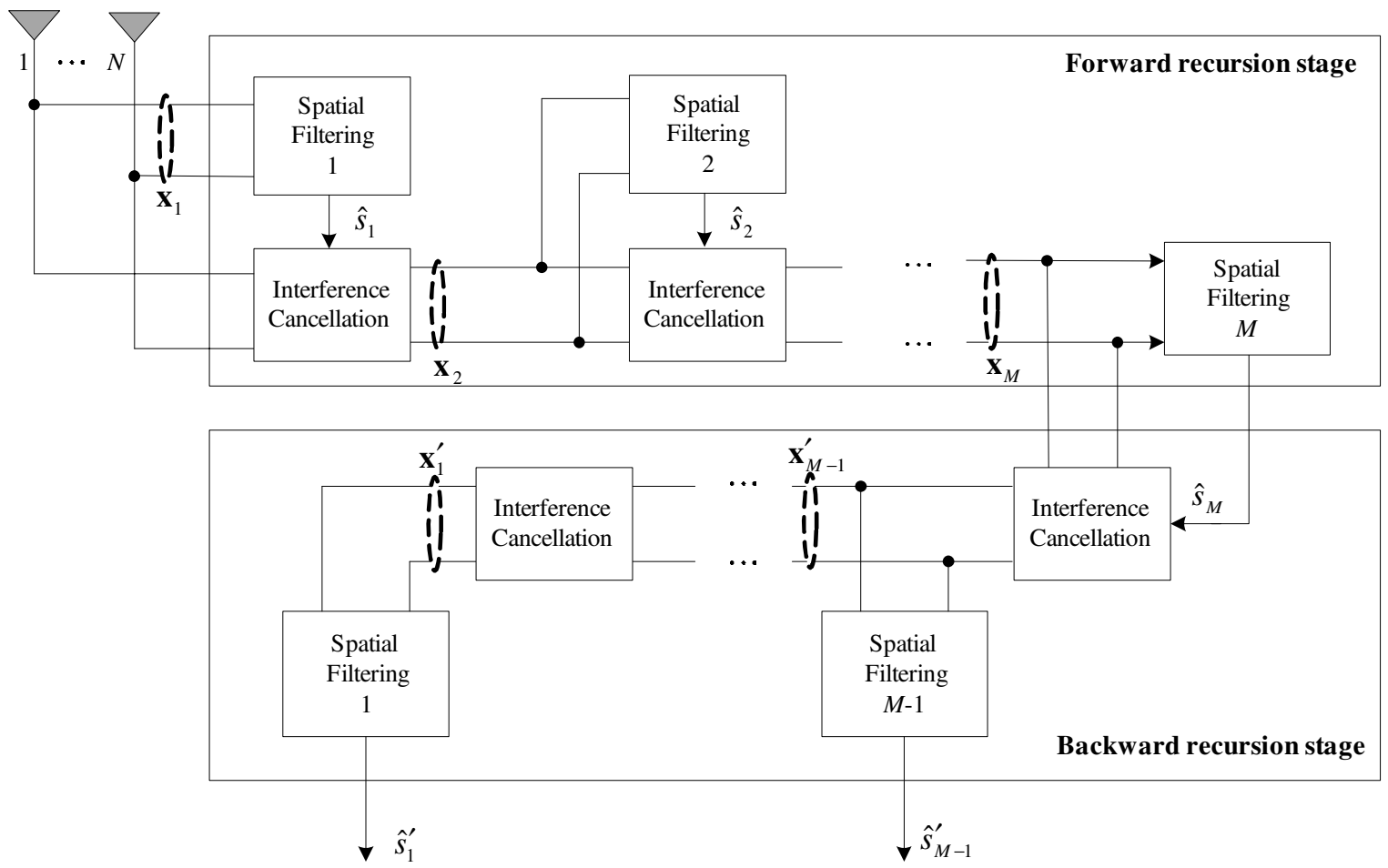

Fig. 1. Structure of SIC receiver with backward recursion processing.

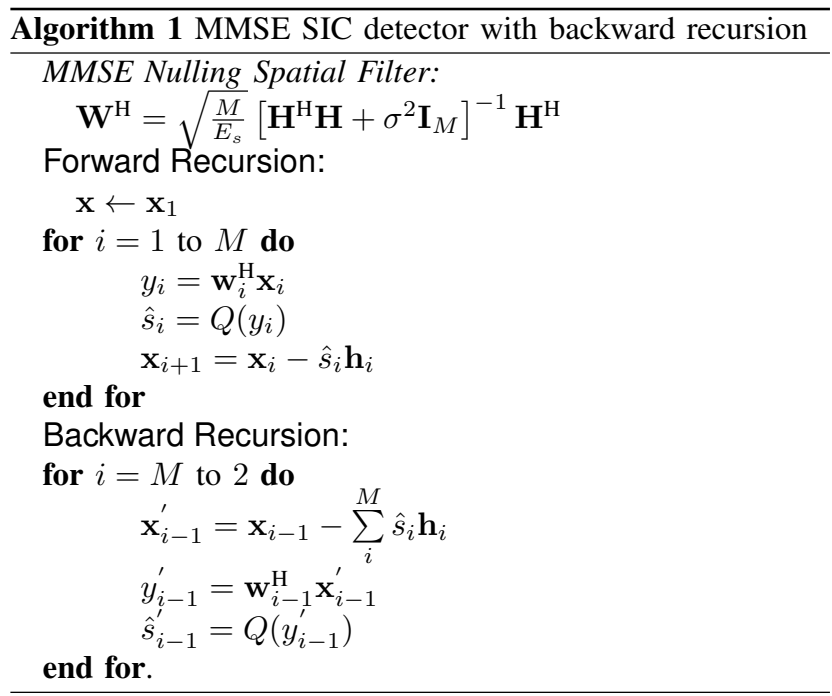

backward recursion stage. Note that, in Figure 1, the forward recursion is the same as classical SIC detection and in this letter we propose an additional step (backward recursion stage) in the end of the traditional approach. The main disadvantage of the traditional SIC-based detector is that only the last layer experiences the full diversity order. To overcome this issue we propose to add the additional stage.

According to the pseudo-code of Algorithm 1 (see also Figure 1), at the $(M-1)$-th backward recursion layer, a modified received signal $\mathbf{x}_{M-1}^{\prime}$ is formed from $\mathbf{x}_{M}$ by subtracting out the interference from the $M$-th layer. A new estimate of the $k$-th symbol transmitted at the $(M-1)$-th layer is then obtained by means of spatial filtering. The backward recursion proceeds similarly for the subsequent layers $M-2, \ldots, 2$. Thanks to this backward recursion stage, now all layers can increase their diversity order.

It is worth mentioning that the proposed backward processing can be extended to ordered SIC (OSIC) detectors [8] by simply adding a detection ordering step in Algorithm 1.

\section{Simulation Results}

In this section we present illustrative simulation results of our proposal. The performance results are evaluated by means of numerical results from Monte-Carlo simulations. The symbol error probability (SEP) curves are plotted against the SNR in $[\mathrm{dB}]$, in this letter we consider that the MIMO system transmits following the traditional VBLAST spatial multiplexing scheme.

Figure 2 presents the SEP performance per layer, in this case we have two spatial multiplexing layers assuming binary phase shift keying (BPSK) modulation and two transmit and two receive antennas. As we can see by the curves from the case without backward recursion, the diversity order is increased in each recursion of the algorithm, thus, providing to the last layer the best SEP performance. Using the backward recursion, now all layers have the same diversity order equal to $N$. Therefore, all two spatial layers have the same SEP performance, corroborating with our claim that with this simple backward recursion all layers are capable to achieve an improved diversity order. It is worth noting that this improvement is achieved without complexity increase since 


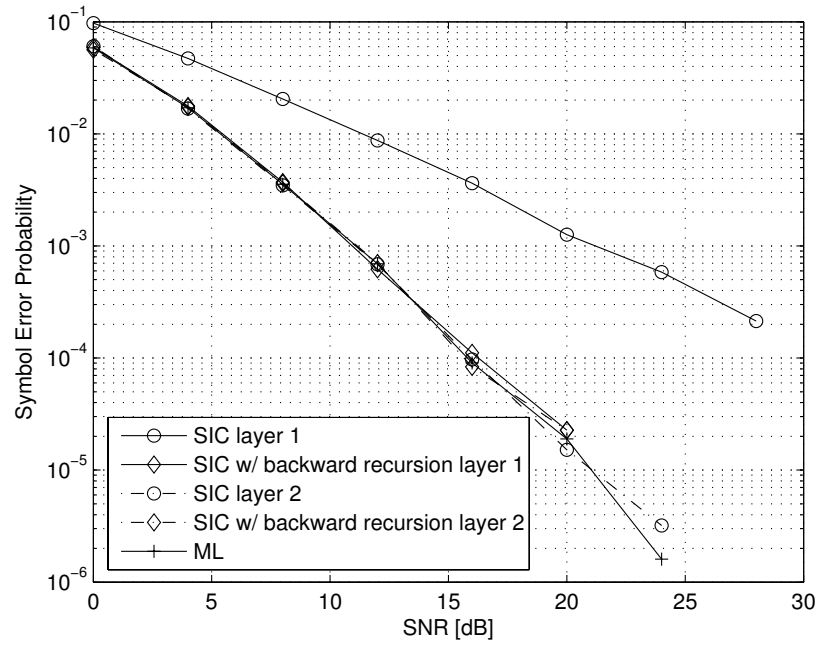

Fig. 2. SEP performance per layer of SIC receiver with and without backward recursion and ML detectors.

the nulling spatial filters are calculated just one time [9]. As a benchmark we included in Figure 2 the result of the maximum likelihood (ML) detector. Since with the backward recursion now all layers reach the full diversity order at the receiver, the performance of the two layers are similar with the ML detector that has a diversity order of $N$.

In Figure 3, we present the comparative result of a VBLAST scheme considering four transmit and four receive antennas using the traditional SIC detector (without backward recursion) and the proposed detector, in both cases with ideal symbol estimation for SIC cancellation assuming quarternary phase shift keying (QPSK) modulation. As a benchmark the performance of the OSIC interference cancellation detector is also considered. Figure 3 presents the comparative performance of the average SEP of all layers. As we can see by results, since now all layers present the same diversity order in the SIC with backward recursion detector, this detector outperforms traditional SIC detector (without backward recursion) and even the SIC with ordering (OSIC). For a SEP of $10^{-3}$, SIC with backward recursion gives an improvement of $5 \mathrm{~dB}$ in the SNR compared with OSIC and almost $15 \mathrm{~dB}$ compared with SIC detector for QPSK modulation.

\section{CONCLUSIONS AND PERSPECTIVES}

In this letter, we have proposed an additional processing stage to be applied in the non-linear interference cancellation detectors, e.g. SIC, denoted as backward recursion. This simple strategy provides to all layers of a spatial multiplexing scheme an improved diversity order at the receiver as evaluated to the VBLAST MMSE SIC detector. Compared with traditional non-linear interference cancellation detectors, our approach provides remarkable SNR gains over traditional SIC and OSIC detectors, respectively. As perspectives we can highlight the extension of this idea to the OSIC detector and the use of an iterative bidirectional forward-backward recursion relying on the turbo processing approach.

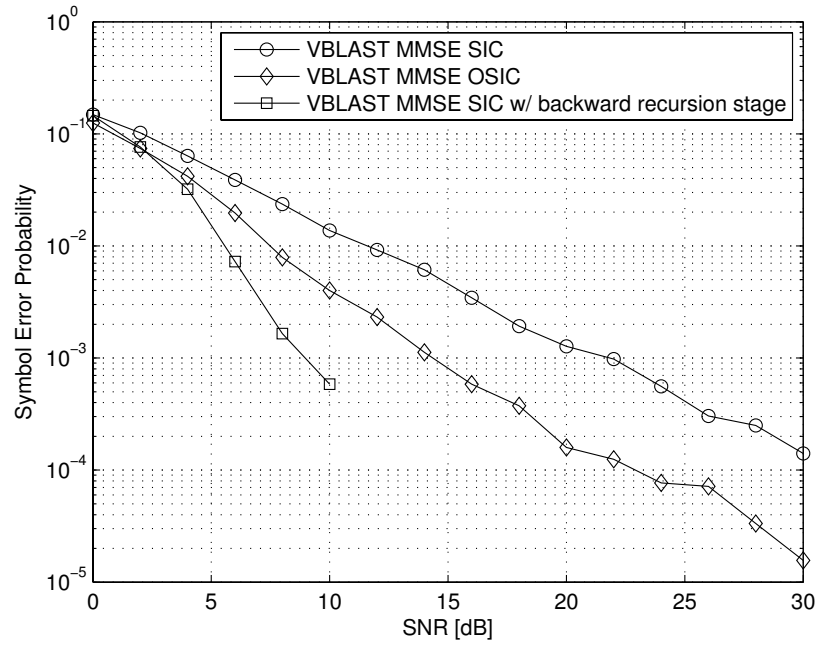

Fig. 3. Average SEP performance comparison of SIC, OSIC and SIC with backward recursion detectors.

\section{REFERENCES}

[1] 3GPP, "Long Term Evolution, Evolved Universal Terrestrial Radio Access (EUTRA); Physical layer; General description," Rel-8, v.8.1.0, Tech. Rep., December 2007.

[2] L. Zheng and D. N. C. Tse, "Diversity and Multiplexing: A Fundamental Tradeoff in Multiple-Antenna Channels," IEEE Transactions on Information Theory, vol. 49, no. 5, pp. 1073-1096, May 2003.

[3] H. Bölcskei, D. Gesbert, C. B. Papadias, and A.-J. van der Veen, Eds., Space-Time Wireless Systems: From Array Processing to MIMO Communications. Cambridge University Press, 2006.

[4] A. J. Paulraj, D. A. Gore, R. U. Nabar, and H. Bölcskei, "An Overview of MIMO Communications A Key to Gigabit Wireless," Proceedings of the IEEE, vol. 92, no. 2, pp. 198-218, February 2004.

[5] A. Sezgin, E. A. Jorswieck, and V. Jungnickel, "Maximum Diversity Detection for Layered Space-Time Codes," in Vehicular Technology Conference, 2003. VTC 2003-Spring. The 57th IEEE Semiannual, vol. 2, April 2003, pp. 833-837.

[6] S. Verdú, Ed., Multiuser detection. Cambridge University Press, 1998.

[7] G. H. Golub and C. F. V. Loan, Eds., Matrix Computations. The Johns Hopkins University Press; 3rd edition (October 15, 1996), 1996.

[8] P. W. Wolniansky, G. J. Foschini, G. D. Golden, and R. A. Valenzuela, "V-BLAST: An Architecture for Realizing Very High Data Rates Over the Rich-Scattering Wireless Channel," Proceedings of the International Symposium on Signals, Systems, and Electronics, pp. 295-300, 1998.

[9] A. Zanella, M. Chiani, and M. Z. Win, "MMSE reception and successive interference cancellation for MIMO systems with high spectral efficiency," IEEE Transactions On Wireless Communications, vol. 4, no. 3, pp. 1244-1253, May 2005.

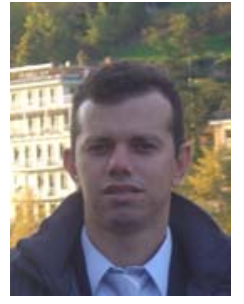

W. C. Freitas Jr. was born on 1976 at Fortaleza, Brazil. He received the B.S., M.S in Electrical Engineering from Federal University of Ceará (UFC) in Fortaleza, Brazil in 2000 and 2002 respectively. In 2006 he obtained his Ph.D. from the Teleinformatic Engineering Department of the Federal University of Ceará (UFC) in Fortaleza, Brazil. Walter was a senior research of Nokia Technology Institute for two years. He is now a professor at UFC and his main area of interest concerns wireless communication systems and the application of link adaptation techniques, MIMO systems and space-time coding. Mr. Freitas is a member of IEEE Communications Society and of the Brazilian Communications Society. 


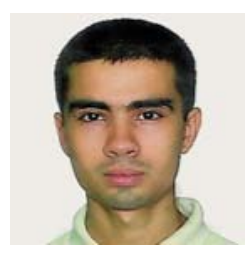

D. C. Moreira Darlan. C. Moreira received Bachelor's degree in electrical engineering and the Master of Science degree in teleinformatics from the Federal University of Ceará (UFC) in 2005 and 2007, respectively. $\mathrm{He}$ is currently pursuing his Doctor's degree at the same institution. He is a member of the Wireless Telecommunications Research Group (GTEL), Fortaleza, Brazil, and since 2004 he has been working in projects within the technical cooperation between GTEL and Ericsson Research. His research interests include link adaptation, MIMO-OFDM, interference alignment.

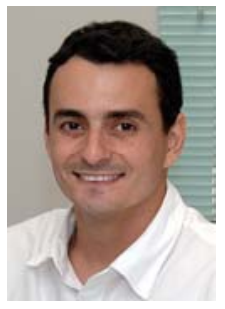

C. C. Cavalcante Charles Casimiro Cavalcante received the B.Sc and M.Sc in Electrical Engineering from the Federal University of Ceará (UFC), Brazil, in 1999 and 2001, respectively, and the D.Sc. degree from the University of Campinas (UNICAMP), Brazil, in 2004. He has held a grant for Scientific and Technological Development from 2004 to 2007 and since March 2009 he has a gran of Scientific Research Productivity both from the Brazilian Research Council (CNPq). From March 2007 to November 2008 he was a visiting professor at Teleinformatics Engineering Department of UFC and since November he is an Assistant Professor at the same department and university holding the Statistical Signal Processing chair. He has been working on signal processing strategies for communications where he has several papers published in journal and conferences, has authored two international patents and he has worked on several funded research projects on the wireless communication area. He is also a co-author of the book Unsupervised Signal Processing: Channel Equalization and Source Separation, published by CRC Press. He is a researcher of the Wireless Telecommunications Research Group (GTEL) where he leads research on signal processing and wireless communications. His main research interests are in signal processing for communications, blind source separation, wireless communications, and statistical signal processing.

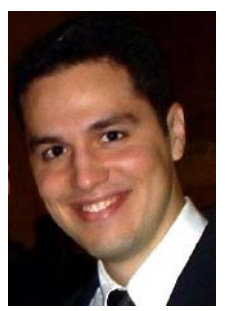

A. L. F. de Almeida André L. F. de Almeida received the B.Sc and M.Sc. degrees in electrical engineering from the Federal University of Ceará, Brazil, in 2001 and 2003, respectively, and the double Ph.D. degree in sciences and teleinformatics engineering from the University of Nice, Sophia Antipolis, France, and the Federal University of Ceará, Fortaleza, Brazil, in 2007. In 2002, he was a Visiting Researcher with Ericsson Research, Stockholm, Sweden. He was a Postdoctoral Fellow with the I3S Laboratory, CNRS, Sophia Antipolis, from January to December 2008. He is currently an Assistant Professor with the Department of Teleinformatics Engineering of the Federal University of Ceará, Fortaleza. He is also a Senior Researcher with the Wireless Telecom Research Group (GTEL), where he has worked on several funded research projects. His research interests lie in the area of signal processing for communications, including blind identification and signal separation, space-time processing, multiple-antenna techniques, and multicarrier communications. His recent work has focused on the development of tensor models for multiple-antenna transceiver design in wireless communication systems. 